(с) М.А. Берковская*, Д.А. Кушханашхова, Ю.П. Сыч, В.В. Фадеев

Первый Московский государственный медицинский университет имени И.М. Сеченова, Москва, Россия

В статье представлен обзор современной литературы о влиянии различных доз витамина D на состояние фосфорно-кальциевого обмена у пациентов после бариатрических операций. Одним из осложнений в послеоперационном периоде является снижение минеральной плотности костной ткани, что приводит к повышенному риску переломов. Существует множество различных механизмов нарушения минерального обмена после бариатрических операций, однако ключевую роль играет снижение всасывания кальция и витамина D. Известно, что витамин D участвует во всех основных процессах, протекающих в костной ткани. Он является наиболее важным эндокринным регулятором кальциевого гомеостаза в организме, обеспечивая всасывание $90 \%$ кальция в кишечнике. У пациентов с морбидным ожирением имеется высокий риск дефицита витамина D еще до проведения операции, который может усугубиться после оперативного вмешательства и при отсутствии своевременного лечения привести к тяжелым нарушениям фосфорно-кальциевого обмена. Обнаружено, что высокие дозы витамина D после бариатрических операций могут способствовать улучшению состояния костного метаболизма и, как следствие, предотвращению развития тяжелых переломов после операции, что в целом благотворно влияет на качество жизни и трудовой прогноз. Результаты имеющихся на сегодняшний день исследований открывают новые возможности для профилактики и лечения послеоперационных осложнений, связанных с нарушением костного обмена.

КЛЮЧЕВЫЕ СЛОВА: витамин D; ожирение; кальчий; бариатрическая операчия; гастрошунтирование; остеопороз.

\title{
CHARACTERISTICS OF CALCIUM AND PHOSPHOROUS METABOLISM IN PATIENTS AFTER BARIATRIC SURGERY AND THE ROLE OF VITAMIN D SUPPLEMENTATION IN THE PREVENTION AND TREATMENT OF POSTOPERATIVE BONE AND MINERAL DISORDERS
}

\author{
(c) Marina A. Berkovskaya*, Dana A. Kushkhanashkhova, Yulia P. Sych, Valentin V. Fadeyev
}

I.M. Sechenov First Moscow State Medical University (Sechenov University), Moscow, Russia

This article provides a review of current literature on the effect of various doses of vitamin D on the parameters of calcium and phosphorus metabolism in patients after bariatric surgery.

The decrease of bone mineral density is one of the most frequent complications of the bariatric surgery, which increases the risk of fractures. There are many different mechanisms for impaired mineral metabolism after bariatric surgery, but a decrease in the absorption of calcium and vitamin D plays a key role in this process. Vitamin D is the most important endocrine regulator of calcium homeostasis in the body, which provides the absorption of $90 \%$ of calcium in the gut. Patients with morbid obesity have a high risk of vitamin D deficiency even before surgery, which may worsen after operation and in the absence of timely treatment lead to severe disturbances of calcium and phosphorus metabolism.

It was found that high doses of vitamin D after bariatric surgery can improve parameters of bone metabolism, and, as a result, prevent fractures after surgery, which generally has a beneficial effect on the quality of life and labor prognosis. The results of the studies available to date open up new opportunities for the prevention and treatment of postoperative complications associated with impaired bone metabolism.

KEYWORDS: vitamin D; obesity; calcium; bariatric surgery; Roux-en-Y; osteoporosis.

\section{АКТУАЛЬНОСТЬ}

Ожирение является эпидемией XXI века. По данным Всемирной организации здравоохранения (ВОЗ), в 2016 г. более 1,9 млрд взрослых старше 18 лет имели избыточный вес. Из них свыше 650 млн страдали ожирением [1]. Ожирение представляется актуальной проблемой со- временной медицины, поскольку приводит к развитию целого ряда тяжелых осложнений: сахарный диабет 2 типа (СД2), сердечно-сосудистые заболевания, дислипидемия, синдром обструктивного апноэ сна, нарушения опорно-двигательной системы, злокачественные опухоли отдельных локализаций, неалкогольная жировая болезнь печени, репродуктивные нарушения и дру- 
гие коморбидные состояния, адекватное лечение которых невозможно без коррекции массы тела. В настоящее время наиболее эффективным методом лечения морбидного ожирения (индекс массы тела (ИМТ) >40 кг/м²) является бариатрическая хирургия. Она существенно сокращает частоту развития сопутствующих ожирению заболеваний и смертность больных. Кроме того, бариатрическая хирургия позволяет существенно снизить финансовые затраты на лечение ожирения и коморбидных состояний [2, 3]. Основными видами бариатрических вмешательств являются рестриктивные и шунтирующие операции, а также их комбинации. К рестриктивным оперативным вмешательствам, приводящим к уменьшению размеров желудка, относят регулируемое бандажирование желудка, вертикальную гастропластику и продольную резекцию желудка. Целью шунтирующих операций является формирование обходного пути, выключающего из функционирования те или иные отделы тонкой кишки и приводящего к мальабсорбции. K мальабсорбтивным операциям относят еюноилеальное и дуоденоеюноилеальное шунтирование, а к комбинированным - гастрошунтирование (ГШ) по Roux-en-Y и билиопанкреатическое шунтирование (БПШ) по HessMarceau [4, 5]. Бариатрические операции, особенно наиболее эффективные (ГШ и БПШ), сопряжены с массивной перестройкой тонкого кишечника, в связи с чем нарушается всасывание кальция и витамина D. Это способствует развитию вторичного гиперпаратиреоза (ВГПТ) и потере минеральной плотности костной ткани (МПК), что в конечном итоге приводит к повышенному риску переломов [6]. Не стоит забывать, что пациенты с морбидным ожирением еще до хирургического лечения имеют дефицит витамина D и ВГПТ, которые могут усугубиться после оперативного вмешательства и при отсутствии своевременного лечения привести к тяжелым нарушениям фосфорно-кальциевого обмена [7].

Согласно клиническим рекомендациям Российской ассоциации эндокринологов по диагностике, лечению и профилактике дефицита витамина D у взрослых, оптимальным уровнем 25(OH)D в крови является $\geq 30$ нг/мл ( $\geq 75$ нмоль/л), что особенно важно для пациентов до и после бариатрических операций, имеющих высокий риск тяжелого дефицита витамина $D$, которым показан биохимический скрининг [8]. В настоящее время не существует единого мнения о форме и режиме дозирования витамина $D$, необходимых для достижения его адекватного уровня с целью профилактики развития ВГПТ и остеопороза после бариатрических операций.

В настоящем обзоре отражены основные механизмы, приводящие к снижению МПК после бариатрических операций, освещена проблема высокой распространенности нарушений костного обмена после данных вмешательств, а также представлены результаты исследований, посвященных изучению влияния различных режимов дозирования и путей введения витамина D на состояние фосфорно-кальциевого обмена и МПК у пациентов после бариатрических операций. Сбор информации для обзора проводился при помощи полнотекстовых баз данных (Springer, eLibrary) и библиографических баз данных (MedLine, Embase, Elsevier) с использованием соответствующих ключевых слов (витамин D, бариатрическая хирургия, ожирение, кальций, гастрошунтирование по Ру, остеопороз, лептин, склеростин, vitamin D, bariatric surgery, obesity, calcium, Roux-en-Y, osteoporosis, leptin, sclerostin), логических операторов (AND NOT OR) и фильтров: 1) типы статей - клиническое исследование, клинический случай, систематический обзор, метаанализ; 2) дата публикаций - последние 10 лет; 3) участники люди. Поиск статистических данных для данного обзора проводился при помощи базы данных HFA DB (European Health for All Database), Who.int.

Механизмы нарушения костного обмена после

бариатрических операций

Помимо нарушения всасывания кальция и витамина D после бариатрических операций, существуют и другие механизмы, лежащие в основе нарушения процессов цикла костного ремоделирования. Они подразделяются на четыре группы и схематично представлены на рисунке 1 [9].

К ним относятся:

1. механическая разгрузка скелета;

2. влияние гормонов жировой ткани;

3. влияние гормонов желудочно-кишечного тракта;

4. пищевые эффекты, заключающиеся в нарушении всасывания микроэлементов.

Bсе механизмы в конечном счете влияют на цикл костного ремоделирования, либо уменьшая процесс образования (остеобластогенез), либо увеличивая процесс разрушения костной ткани (остеокластогенез).

1. Механическая разгрузка скелета. Известно, что остеоциты - клетки костной ткани, которые воспринимают любые изменения упругого напряжения костной ткани и трансформируют механические стимулы в биохимические сигналы, инициируя процессы ремоделирования в определенном локусе [10]. Механическая нагрузка, воспринимаемая остеоцитами, способствует снижению продукции склеростина. Склеростин является наиболее чувствительным костно-специфическим антагонистом Wnt-сигнального пути [11]. Wnt-сигнальный путь играет ключевую роль в регуляции остеобластогенеза, активация которого происходит путем связывания Wnt (винглес)-белка с рецептором фризельда (ФРЗ) и его корецептором - белком, связанным с липопротеидами низкой плотности 5 и 6 (LRP5, -6). Данное взаимодействие способствует переходу $\beta$-катенина в ядро клетки, активируя факторы транскрипции TCF/LEF (транскрипционный фактор / лимфоидный энхансер-связывающий фактор), что в конечном итоге приводит к дифференцировке мезенхимальной стволовой клетки в остеобласт, а также к выживанию и продолжению дифференцировки преостеобластов в зрелые клетки с последующим увеличением интенсивности костеобразования. При механической разгрузке скелета после операции увеличивается экспрессия склеростина остеоцитами. Склеростин образует связь с LRP5 (рецепторы липопротеидов низкой плотности 5) на поверхности клетки, нарушая взаимодействие Wnt-белка с рецептором, блокируя остеобластогенез [11].

Таким образом, механическая разгрузка приводит к потере костной массы за счет подавления остеобластогенеза. В качестве одной из перспективных целей терапевтического вмешательства возможно рассмотреть склеростин, блокирование которого моноклональными 


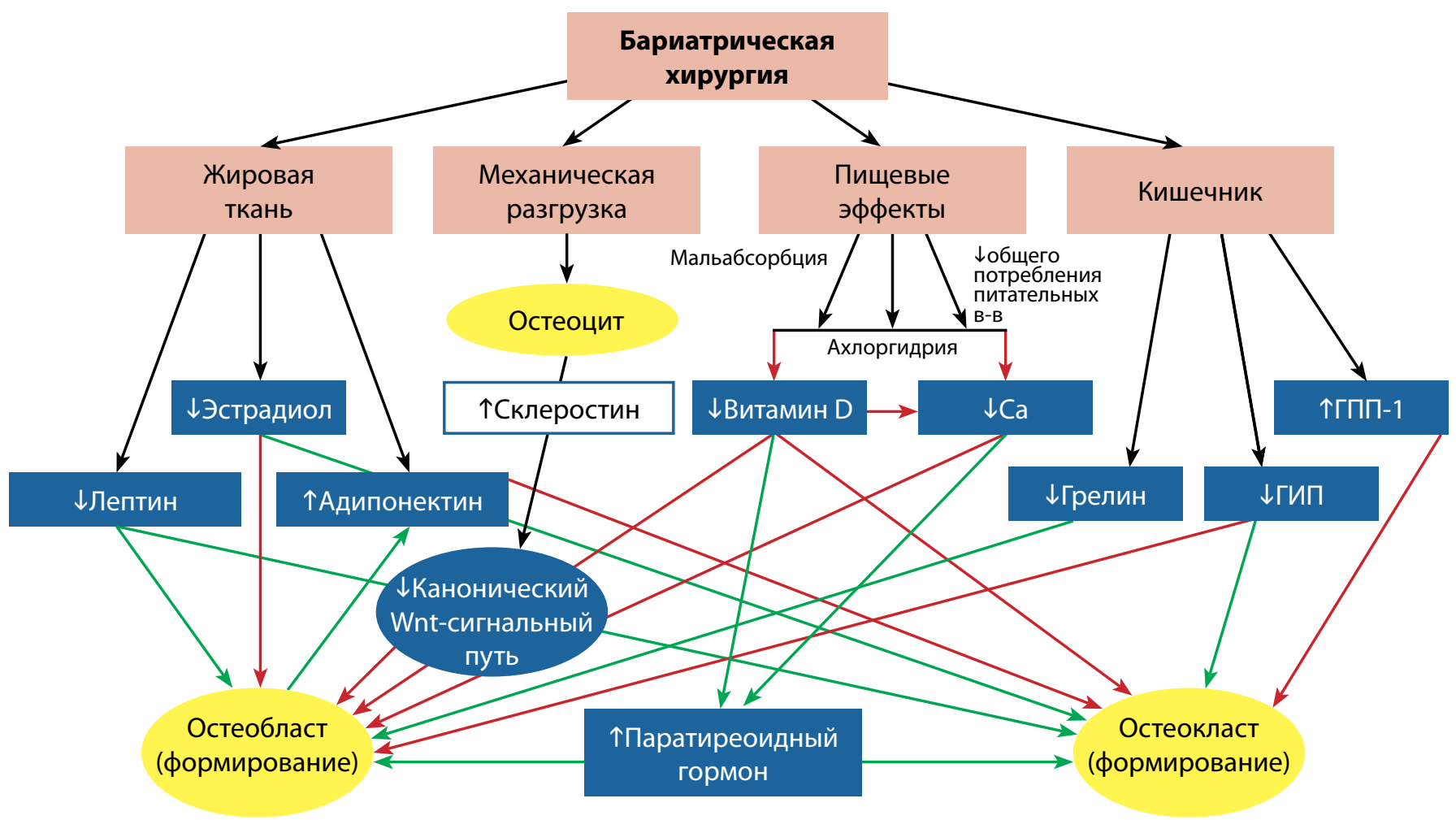

Рисунок 1. Механизмы нарушения костного обмена (адаптировано из Folli F, et al. 2012 [9])

Примечание: красные стрелки указывают на ингибирование резорбции/образования, зеленые стрелки указывают на стимуляцию резорбции/ образования

антителами оказывает анаболический эффект на костную ткань, предотвращая потерю костной ткани после бариатрических операций.

2. Влияние гормонов жировой ткани. Гормоны жировой ткани также являются кандидатами на участие в регуляции метаболизма костной ткани. После бариатрических операций вследствие снижения массы жировой ткани происходит изменение секреции адипокинов (лептина и адипонектина) и снижение уровня эстрадиола (за счет снижения ароматизации тестостерона) [9]. Эстрадиол стимулирует синтез остеопротегерина, который, связывая RANKL (лиганд рецептора ядерного фактора кB), блокирует остеокластогенез. Таким образом, при снижении уровня эстрогенов усиливается процесс резорбции костной ткани $[10,11]$.

Лептин - гормон жировой ткани, который информирует мозг о запасах энергии в организме. Помимо этого, лептин оказывает положительное влияние на костную ткань путем стимуляции дифференцировки остеобластов и угнетения остеокластогенеза [12]. Уровень лептина значительно снижался после бариатрической операции и обратно коррелировал с уровнем маркеров костеобразования и резорбции. Согласно данным Bruno С. и соавт., увеличение маркеров резорбции (N-концевой телопептид коллагена I типа) значительно превышало изменение маркеров костеобразования, что свидетельствовало о преобладании процессов разрушения костной ткани [13].

Адипонектин, так же как и лептин, продуцируется жировой тканью. Его рецепторы были обнаружены как на остеобластах, так и на остеокластах, при стимуляции которых усиливается процесс дифференцировки остеобластов и одновременно подавляются образование и активность остеокластов [14]. У пациентов после бариатрических операций уровень адипонектина значительно увеличивается, оказывая положительное влияние на костный обмен [9].

3. Влияние гормонов желудочно-кишечного тракma. Одним из механизмов, влияющих на изменение процессов костного ремоделирования, является изменение секреции кишечных гормонов.

Глюкагоноподобный пептид-1 (ГПП-1) и глюкозозависимый инсулинотропный пептид (ГИП) оказывают положительное влияние на костный обмен. ГИП способствует активации остеобластогенеза и подавляет остеокластогенез $[15,16]$. ГПП-1 воздействует на стромальные клетки, вызывает клеточную пролиферацию и направляет клеточную дифференцировку по пути остеобластов, предотвращая дифференцировку в адипоциты [17]. Таким же образом на костную ткань воздействует еще один гормон желудочно-кишечного тракта (ЖКТ) - грелин. После бариатрических операций наблюдалось снижение уровней ГИП и грелина, что отрицательно влияло на костный обмен $[18,19]$. В свою очередь, уровень ГПП-1 после шунтирующих операций увеличивался, оказывая положительное влияние на костный обмен, потенцируя процесс формирования костной ткани [20].

4. Мальабсорбтивные изменения. Одним из важных макроэлементов, всасывание которого нарушается после бариатрических операций, является кальций. Существует два механизма абсорбции кальция в кишечнике: насыщаемый трансклеточный путь и ненасыщаемый параклеточный транспорт [21]. Наиболее изученный механизм транспорта кальция в кишечнике - трансклеточный - зависим от 1,25(OH), D. Он индуцирует экспрессию клаудина-2 и клаудина-12, которые связаны с абсорбцией 
кальция в кишечнике и формируют параклеточные каналы между прилежащими клетками. Данный путь включает три этапа: поступление кальция в энтероцит, транспорт кальция в клетке и перемещение его через базолатеральную мембрану во внеклеточную жидкость.

$1,25(\mathrm{OH})_{2}$ D запускает синтез различных белков щеточной каймы, включая интестинальный кальций-связывающий белок, щелочную фосфатазу щеточной каймы и низкоаффинную $\mathrm{Ca}^{2+} / \mathrm{Mg}^{2+}$-АТФазу. В двенадцатиперстной и тощей кишке, а также почках и других тканях экспрессируются TRPV5 и TRPV6 - два вида кальциевых каналов, относящихся к подсемейству ванилоидных рецепторов транзиторного потенциала и содержащих шесть трансмембранных доменов. TRPV6 особенно важен для кишечной абсорбции кальция, а 1,25(OH), D увеличивает его экспрессию, как и экспрессию TRPV5, который отвечает за реабсорцию кальция в почках. При поступлении в энтероцит кальций связывается с компонентами комплекса щеточной каймы, подлежащего цитоплазматической мембране. В ответ на действие $1,25(\mathrm{OH})_{2} \mathrm{D}$ кальмодулин перераспределяется к щеточной кайме и может участвовать в этом процессе, как и индуцируемый 1,25(OH), D кальций-связывающий белок кальбиндин-9К (рис. 2) [22].

Запуск синтеза кишечного кальций-связывающего белка кальбиндина-9К - наиболее изученный эффект витамина D в отношении энтероцитов. Этот белок имеет структуру типа EF-hand, что обеспечивает связывание одной молекулой двух ионов кальция. Сродство кальбиндина к кальцию приблизительно в 4 раза сильнее, чем у кальций-связывающего компонента щеточной каймы, вследствие чего кальций перемещается в связь с кальбиндином. Кальбиндин служит буфером внутриклеточного свободного кальция в процессе его абсорбции. Он связывается с микротрубочками и способен участвовать в транспорте кальция внутри энтероцита. Перенос кальция через базальную поверхность энтероцита - последний процесс, участвующий в кишечной абсорбции кальция, - зависит от 1,25(OH) 2 D. Основным механизмом выведения кальция служит индуцируемый 1,25(OH) 2 D АТФ-зависимый кальциевый насос (PMCA1b кальций-транспортирующая АТФаза плазматической мембраны типа 1b). Сродство насоса к кальцию приблизительно в 2,5 раза выше, чем у кальбиндина [21].

Таким образом, витамин D создает надлежащую микросреду для минерализации костной ткани путем стимуляции абсорбции кальция в кишечнике. Так как витамин D является жирорастворимым витамином, для его усвоения необходимы определенное $\mathrm{pH}$ кишечника, желчные кислоты и ферменты поджелудочной железы. После бариатрических операций происходят выраженная анатомическая перестройка ЖКТ, резкое ограничение объема поступающей пищи в желудок, теряется прямая стимуляция пищей секреции соляной кислоты, развивается мальабсорбция, увеличивается транзит через кишечник и изменяется метаболизм желчных кислот [23]. Это способствует изменению привычных условий для всасывания кальция и витамина D в ЖКТ. Все это приводит к нарушению процессов минерализации костной ткани, что является одним из важных механизмов развития остеопороза.

В одном из последних исследований уделяется особое внимание гранулоцитарному колониестимулирующему фактору (Г-КСФ), который увеличивается после операции вертикальной гастропластики (ВГП) у мышей и людей. В мышиной модели операция способствовала потере костной ткани, которая не зависела от пола, массы тела и диеты, и эта потеря характеризовалась нарушением минерализации и формирования костной ткани, что было связано с повышением уровня Г-КСФ после операции. То же самое наблюдалось и у людей транзиторное повышение уровня Г-КСФ через 1 и 3 мес после операции ВГП и возвращение к исходному уровню через 6 и 12 мес, однако оценка МПК у пациентов не проводилась. В предыдущих исследованиях было выявлено, что Г-КСФ ингибирует функцию остеобластов и нарушает формирование костной ткани, стимулирует миелопоэз и увеличивает количество циркулирующих нейтрофилов $[24,25]$. Таким образом, это еще один потенциальный механизм, воздействие на который, возможно, будет способствовать предотвращению тяжелых осложнений костного обмена, однако многое в этом вопросе еще остается предметом будущих исследований.

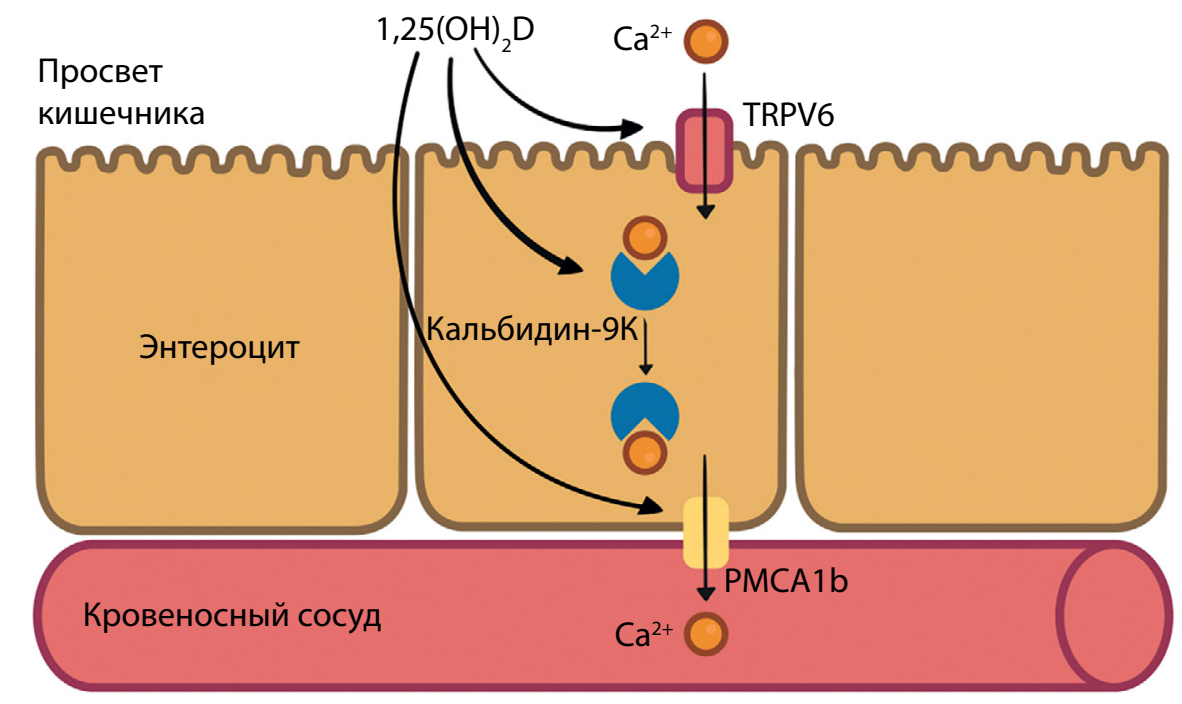

Рисунок 2. Механизм всасывания кальция в кишечнике (Адаптировано из Corbeels K, et al. 2018 [22]).

Аббревиатуры: TRPV6 - кальциевый канал; PMCA1b - кальций-транспортирующая АТФаза плазматической мембраны типа 1b 
Распространенность нарушений костного метаболизма после бариатрических операций

Патогенез снижения МПК после бариатрических операций сложен и не ограничивается только нарушением всасывания кальция и витамина D. Актуальность данной проблемы подчеркивается крайне высокой распространенностью послеоперационных нарушений фосфорно-кальциевого обмена у лиц, подвергшихся бариатрическим вмешательствам. На сегодняшний день опубликовано большое количество работ как отечественных, так и зарубежных авторов, посвященных изучению этой проблемы.

Так, в целом ряде работ показано значимое повышение распространенности дефицита витамина D и ВГПТ у пациентов после бариатрических вмешательств, по сравнению с лицами с нормальной массой тела, ожирением и ИМТ <40 кг/м², пациентами с морбидным ожирением до оперативного лечения, а также продемонстрировано снижение МПК после операций [26-29]. Следует отметить, что формирующиеся нарушения фосфорно-кальциевого обмена, характерные для данной категории пациентов, носят, по всей видимости, стойкий характер и при отсутствии адекватной коррекции сохраняются в течение длительного времени. Так, в 2014 г. Karefylakis C. оценивал распространенность дефицита витамина D и ВГПТ спустя 10 лет после ГШ. Было обследовано 293 пациента, 83\% из которых - женщины в возрасте 49+9,9 года. Уровни 25(OH)D, ПТГ и альбуминскорректированные значения кальция составили

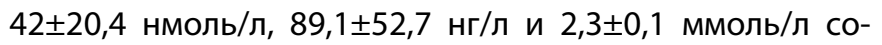
ответственно. Из всех пациентов 65\% имели дефицит витамина D (25(OH)D <50 нмоль/л) и 69\% - повышение уровня ПТГ выше верхней границы референсного диапазона (>73 нг/л). Единственным фактором, способным предсказать дефицит витамина D до операции, был высокий дооперационный ИМТ $(p=0,008)$. Результаты этого исследования вызывают тревогу и подчеркивают необходимость улучшения долгосрочного наблюдения за такими пациентами [30].

Для оценки нарушения цикла костного ремоделирования у пациентов с тяжелой степенью ожирения, перенесших бариатрические операции, в ряде исследований, помимо кальция и 25(OH)D, оценивались также маркеры костного метаболизма. В исследовании Svanevik M. у пациентов спустя 2 года после операции ГШ было выявлено повышение уровня маркеров костного обмена (СТX - С-концевого телопептида коллагена I типа, P1NP - N-терминального пропептида проколлагена I типа, щелочной фосфатазы) [31]. Аналогичные результаты были выявлены в предыдущих исследованиях $[26,32,33]$.

В исследовании Muller M.K. также было выявлено ускорение цикла костного обмена после бариатрических операций, причем с преобладанием резорбции костной ткани. В качестве маркера авторы использовали соотношение P1NP/CTX. У 114 пациентов были взяты анализы крови до операции, через 1 мес и через 1 год после ГШ. Через 1 год после операции соотношение P1NP/CTX значительно уменьшилось по сравнению с исходным уровнем (в среднем на 27\%), что говорит об ускорении костного ремоделирования с преобладанием резорбции костной ткани после операции [34].
Значимость контроля за состоянием костного метаболизма после бариатрической хирургии подтверждается множеством описанных случаев низкотравматичных переломов костей позвоночника и проксимального отдела бедра через несколько лет после проведения данных операций [35, 36, 37].

В 2018 г. был описан случай с развитием множественных переломов позвонков уже спустя 5 мес после операции ГШ [38]. Пациентка, 40 лет, спустя 5 мес после операции потеряла 55 кг от исходной массы тела, по результатам анализов был выявлен ВГПТ. Это был первый описанный случай переломов спустя такой короткий срок после операции.

Учитывая все вышесказанное, напрашивается закономерный вывод о том, насколько важно уделять внимание состоянию костного обмена, начиная с самых ранних этапов послеоперационного периода и в течение всего времени наблюдения за пациентами после бариатрического лечения.

Пути коррекции костных нарушений после

бариатрических операций

Учитывая высокую распространенность дефицита витамина D и ВГПТ у пациентов после бариатрических операций, а также важную роль этих нарушений в снижении МПК и повышении риска переломов, неудивительно, что назначение витамина D и препаратов кальция является наиболее распространенным и изучаемым подходом к коррекции послеоперационных костных нарушений. Тем не менее до сих пор единых режимов дозирования и схем назначения витамина $\mathrm{D}$ в данной категории пациентов не разработано, это является предметом активного изучения.

В настоящее время опубликован ряд исследований, изучающих влияние различных доз и путей введения витамина D на состояние костного обмена у пациентов после бариатрических операций.

Одной из таких работ является рандомизированное клиническое исследование Goldner W.S., в котором оценивались различные дозировки витамина D (800, 2000 и 5000 ME/сут) у пациентов, перенесших операцию ГШ. В исследовании участвовали 45 пациентов, у которых в сыворотке крови определяли 25(OH)D, интактный паратгормон (ПТГ), кальций и соотношение кальция и креатинина в моче через 6, 12 и 24 мес после операции. Было выявлено, что наибольшее увеличение уровня 25(OH)D наблюдалось в группе, которая получала 5000 ME/сут [39].

В другом исследовании того же года Carlin A.M. и соавт. использовали другие дозы витамина D. Оценивались параметры костного обмена и МПК. В исследование были включены 60 женщин с дефицитом витамина $D$, которые после операции ГШ были рандомизированы на 2 группы. Первая группа $(\mathrm{n}=30)$ получала per os 50000 ME каждую неделю и дополнительно витамин D 800 ME каждый день, а также кальций 1500 мг/сут, 2-я группа ( $\mathrm{n=30}$ ) принимала только ежедневную добавку 800 ME витамина D и 1500 мг кальция. У обеих групп до операции и через год после нее был взят анализ крови на содержание кальция, ПTГ, 25(OH)D, костноспецифической щелочной фосфатазы и $\mathrm{N}$-телопептида в моче, а также оценена МПК. Через 1 год после ГШ в 1-й группе 
средний уровень 25(OH)D был достоверно выше, чем во 2-й $(p<0,001)$. Никаких различий по уровню ПТГ между группами выявлено не было. МПК проксимального отдела бедра спустя год после ГШ была снижена в обеих группах ( $<<0,001)$, но процент снижения МПК бедра В 1-й группе был на 33\% меньше, чем во 2-й $(p=0,043)$. Из этого следует, что назначение доз 50000 ME еженедельно после ГШ безопасно корректирует дефицит витамина D у большинства женщин, а также способствует ослаблению потери МПК [40].

В исследовании Maria Luger и соавт. изучали эффективность и безопасность режима форсированного дозирования витамина D у пациентов с ожирением и дефицитом витамина D, планирующих операцию омега-петлевое ГШ (модификация лапароскопического ГШ с формированием позадиободочного гастроеюноанастомоза (ОЛГБ)) [41, 42]. Это было двойное слепое рандомизированное, плацебо-контролируемое исследование, в котором принимали участие 50 пациентов с дефицитом витамина D, планирующих ОЛГБ. Пациенты были случайным образом распределены на две группы по 25 человек. Первой группе (группе вмешательства) была назначена нагрузочная доза 300000 ME витамина D3 (холекальциферол) per os, разделенная на три дозы (каждая по 100000 ME). Первая нагрузочная доза - на 1-й или 2-й день после операции, затем через 2 нед и третья доза - на 4-й неделе после операции. После чего назначалась поддерживающая доза в размере 3420 ME/сут (приблизительно 24000 МЕ/нед). Все пациенты в группе вмешательства получали первую нагрузочную дозу. Вторая и третья дозы назначались, если 25(OH)D не достигал уровня 75 нмоль/л, в таком случае снова назначалась доза 100000 ME, и так далее до 300000 ME). Вторая группа (контрольная) получала плацебо в качестве нагрузочной дозы и затем поддерживающую дозу, так же как и группа вмешательства. Далее в течение 24 нед пациенты подлежали тщательному наблюдению и обследованию, которое включало в себя биохимический анализ крови, анализ крови на 25(OH)D, маркеры костного метаболизма, маркеры воспаления и инсулинорезистентности, биопсию печени, состояние микробиоты кишечника и оценку состояния МПК по данным денситометрии.

По результатам исследования было выявлено достоверное увеличение уровня витамина D в течение всего периода наблюдения $(p<0,001)$ с наибольшей концентрацией в 1-й группе по сравнению с контрольной $(p=0,046)$. Концентрация кальция в сыворотке крови оставалась стабильной на протяжении всего исследования в каждой группе. Значительное подавление ПТГ было выявлено исключительно в группе вмешательства, в связи с чем группа вмешательства имела достоверно более низкую распространенность и частоту развития ВГПТ по сравнению с контрольной группой. Таким образом, полученные данные свидетельствуют о том, что начало приема витамина D именно с такой высокой нагрузочной дозы в первый месяц послеоперационного периода с последующей поддерживающей дозой является эффективным и безопасным в достижении более высоких концентраций 25(OH)D у пациентов, перенесших операцию омега-петлевое шунтирование желудка, что способствует предотвращению развития отдаленных последствий со стороны костного обмена.
В одном из последних исследований 2019 г. также сравнивались два различных режима приема холекальциферола [43]. В исследование были включены 100 пациентов, которые перенесли рукавную резекцию желудка или желудочное шунтирование в период с июня 2015 г. по январь 2016 г. Пациенты были распределены на две группы: Группа A $(\mathrm{n}=50)$ получала 1000 мг кальция и $800 \mathrm{ME}$ холекальциферола per os. Группа Б $(\mathrm{n}=50)$ получала 1000 мг кальция и $800 \mathrm{ME}$ холекальциферола, а также 1 мл жидкого холекальциферола (50 $000 \mathrm{ME}$ ) ежемесячно. Оценивались следующие показатели костного обмена: уровень кальция, ПТГ и 25(OH)D до операции и через 3 и 6 мес после нее. В случае выявления дефицита витамина D до операции пациентам назначали холекальциферол per os (50 $000 \mathrm{ME}) 1$ раз в неделю в течение первых 6 нед, а затем 50000 ME ежемесячно. Из 100 пациентов дефицит витамина D до операции был выявлен у 91 - 47 из группы А и 44 из группы В. После операции наблюдалось повышение уровня 25(OH)D в обеих группах

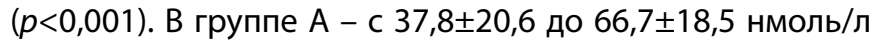
через 6 мес после операции $(p<0,001)$. В группе $\mathrm{B}$ -

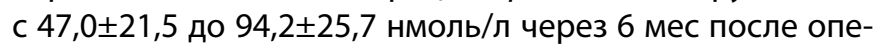
рации $(p<0,001)$. В каждой из групп были пациенты, по-прежнему имеющие дефицит витамина D: в группе A - 35 пациентов, в группе B - 10 пациентов. Никаких различий по уровню кальция в группе $A(p=0,058)$ и группе $Б(p=0,930)$ не наблюдалось. Снижение уровня ПтГ отмечалось в обеих группах, в группе A - с 7,5 $\pm 3,1$ до 6,5 2,9 пмоль/л через 6 мес после операции $(\mathrm{p}<0,032)$

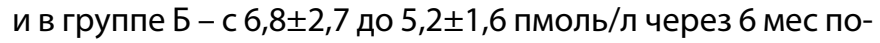
сле операции $(p<0,001)$. Достоверных различий по уровню 25(OH)D $(p=0,851)$, кальция $(p=0,080)$, ПТГ $(p=0,482)$ между пациентами, которые перенесли рукавную резекцию желудка или желудочное шунтирование до и через 6 мес после операции, выявлено не было.

Таким образом, использование дозировки $800 \mathrm{ME}$ холекальциферола ежедневно не было достаточным для восстановления уровня 25(OH)D у 35 из 50 пациентов. Стандартный режим приема $800 \mathrm{ME}$ с добавлением $50000 \mathrm{ME}$ холекальциферола ежемесячно показал более заметное снижение количества пациентов с дефицитом витамина D, однако 10 из 50 пациентов по-прежнему имели дефицит [44].

Возможно, даже такой режим дозирования слишком мал для данной когорты пациентов. Как уже было сказано выше, до сих пор не существует единого мнения об адекватной дозе витамина $\mathrm{D}$ для пациентов после бариатрической хирургии, в связи с чем очевидной является необходимость дальнейших исследований в данном направлении.

Важность восполнения дефицита витамина D и кальция после бариатрического лечения подчеркивается также тем фактом, что данные нутриенты, возможно, могут влиять не только на ось паращитовидные железы/костная ткань, но и затрагивать другие патогенетические пути снижения МПК после операции. Например, в исследовании Muschitz C. и соавт. было показано, что назначение препаратов кальция, витамина D и белка вместе с физической нагрузкой у пациентов после бариатрических операций приводит к меньшему повышению уровня склеростина и маркеров костного ремоделирования СTX и P1NP по сравнению с контрольной группой 
пациентов без вмешательств [45]. Тем не менее, несмотря на положительное влияние указанных вмешательств на показатели костного метаболизма, удельный вклад каждого из них (витамин D, кальций, белок, физическая нагрузка) остается неясным.

Очевидно, что для разработки алгоритма коррекции костных нарушений у пациентов после бариатрических вмешательств необходимы долгосрочные проспективные исследования с большим размером выборки, включающие различные режимы дозирования и схемы приема витамина D и кальция и оценивающие распространенность заболеваний костной ткани и риск переломов после бариатрических операций.

\section{ЗАКЛЮЧЕНИЕ}

В настоящее время бариатрические операции являются эффективным методом лечения ожирения. Однако данные вмешательства сопряжены с рядом осложнений, одним из которых является мальабсорбция, приводящая к дефициту витамина D и в последующем - потере МПК и развитию остеопороза. В связи с этим необходимо комплексное обследование пациентов на дооперационном этапе и последующее тщательное динамическое наблю- дение после операции. Помимо определения уровня кальция, ПТГ и 25(OH)D, может являться целесообразным дополнительное исследование уровня маркеров костного обмена, а также оценка МПК по данным денситометрии. Результаты последних исследований показывают безопасность режима нагрузочных доз витамина $\mathrm{D}$ для поддержания его нормального уровня в крови и, возможно, предотвращения развития осложнений, ассоциированных с его дефицитом. Тем не менее, многое в этом вопросе еще является неопределенным и требует дальнейшего изучения с целью профилактики развития тяжелых осложнений со стороны костного метаболизма и выработки оптимальных терапевтических стратегий.

\section{ДОПОЛНИТЕЛЬНАЯ ИНФОРМАЦИЯ}

Источник финансирования. Подготовка и публикация рукописи проведены на личные средства авторского коллектива.

Конфликт интересов. Авторы декларируют отсутствие явных и потенциальных конфликтов интересов, связанных с публикацией настоящей статьи.

Участие авторов. Все авторы внесли значимый вклад в проведение исследования и подготовку статьи, прочли и одобрили финальную версию статьи перед публикацией.

\section{СПИСОК ЛИТЕРАТУРЫ | REFERENCES}

1. Who.int [Internet]. Obesity and overweight [updated 2020 Mar 3; cited 2020 Apr 15]. Available from: https://www.who.int/news-room/ fact-sheets/detail/obesity-and-overweight.

2. Дедов И.И., Мельниченко Г.А., Шестакова М.В., и др. Национальные клинические рекомендации по лечению морбидного ожирения у взрослых. 3-ий пересмотр (лечение морбидного ожирения у взрослых). // Ожирение и метаболизм. - 2018. - Т. 15. №1. - C. 53-70. [Dedov II, Mel'nichenko GA, Shestakova MV, et al. Russian national clinical recommendations for morbid obesity treatment in adults. 3rd revision (Morbid obesity treatment in adults). Obesity and metabolism. 2018;15(1):53-70. (In Russ.)] doi: https://doi.org/10.14341/OMET2018153-70

3. Колешко С.В., Дубровщик О.И., Мармыш Г.Г., Довнар И.С. Хирургические методы лечения ожирения: реалии и возможности на современном этапе. // Журнал Гродненского государственного медицинского университета. — 2016. — №2. C. 5-9. [Koleshko SV, Dubrovshchik Ol, Marmysh GG, Dovnar IS. Surginal treatments obesity: realitiesand capabilities for today. Zhurnal Grodnenskogo gosudarstvennogo meditsinskogo universiteta. 2016;(2):5-9. (In Russ.)]

4. Салухов В.В., Блэк М.С., Барсуков А.В, и др. Перспективы бариатрических вмешательств у пациентов с метаболическим синдромом. // Concilium medicum. - 2017. - T. 19. №10. - C. 123-131. [Salukhov W, Black MS, Barsukov AV, et al. Prospects of bariatric interventions in patients with metabolic syndrome. Consilium medicum. 2017;19(10):123-131. (In Russ.)] doi: https://doi.org/10.26442/2075-1753_19.10.123-131

5. Окороков П.Л., Васюкова О.В., Дедов И.И. Бариатрическая хирургия в лечении морбидного ожирения у подростков (обзор литературы). // Проблемы эндокринологии. - 2016. - Т. 62. №3. - C. 25-32. [Okorokov PL, Vasyukova OV, Dedov II. Bariatric surgery in the treatment of morbid obesity in adolescents (literature review). Problems of endocrinology. 2016;62(3):25-32. (In Russ.)] doi: https://doi.org/10.14341/probl201662325-32

6. Corbeels $\mathrm{K}$, Verlinden $\mathrm{L}$, Lannoo M, et al. Thin bones: Vitamin D and calcium handling after bariatric surgery. Bone Rep. 2018;8:57-63. doi: https://doi.org/10.1016/j.bonr.2018.02.002

7. Fish E, Beverstein G, Olson D, et al. Vitamin D status of morbidly obese bariatric surgery patients. J Surg Res. 2010;164(2):198-202. doi: https://doi.org/10.1016/j.jss.2010.06.029

8. Пигарова Е.А., Рожинская Л.Я., Белая Ж.Е., и др. Клинические рекомендации Российской ассоциации эндокринологов по диагностике, лечению и профилактике дефицита витамина D у взрослых. // Проблемы эндокринологии. - 2016. T. 62. - №4. - C. 60-84. [Pigarova EA, Rozhinskaya LY, Belaya JE, et al. Russian Association of Endocrinologists recommendations for diagnosis, treatment and prevention of vitamin D deficiency in adults. Problems of endocrinology. 2018;62(4):60-84. (In Russ.)] doi: https://doi.org/10.14341/probl201662460-84

9. Folli F, Sabowitz BN, Schwesinger W, et al. Bariatric surgery and bone disease: from clinical perspective to molecular insights. Int J Obes (Lond). 2012;36(11):1373-1379. doi: https://doi.org/10.1038/ijo.2012.115

10. Мкртумян А.М., Бирюкова Е.В., Егшатян Л.В. Остеопороз. Учебно методическое пособие для врачей, клинических ординаторов и интернов. - М.: Издательство АМИ Медфорум; 2018. [Mkrtumyan AM, Biryukova EV, Egshatyan LV. Osteoporoz. Uchebnometodicheskoe posobie dlya vrachey, klinicheskikh ordinatorov i internov. Moscow: AMI Medforum,2018. (In Russ.)]

11. Гребенникова Т.А., Белая Ж.Е., Рожинская Л.Я., и др. Эпигенетические аспекты остеопороза. // Вестник РАМН. 2015. — T. 70. — №5. - C. 541-548. [Grebennikova TA, Belaya ZE, Rozhinskaya LY, et al. Epigenetic Aspects of Osteoporosis. Annals of the Russian academy of medical sciences. 2015;70(5):541-548. (In Russ.)] doi: https://doi.org/10.15690/vramn.v70.i5.1440

12. Gordeladze JO, Drevon CA, Syversen U, Reseland JE. Leptin stimulates human osteoblastic cell proliferation, de novo collagen synthesis, and mineralization: Impact on differentiation markers, apoptosis, and osteoclastic signaling. J Cell Biochem. 2002;85(4):825-836. doi: https://doi.org/10.1002/jcb.10156

13. Bruno C, Fulford AD, Potts JR, et al. Serum markers of bone turnover are increased at six and 18 months after Rouxen-Y bariatric surgery: correlation with the reduction in leptin. J Clin Endocrinol Metab. 2010;95(1):159-166. doi: https://doi.org/10.1210/jc.2009-0265

14. Oshima K, Nampei A, Matsuda M, et al. Adiponectin increases bone mass by suppressing osteoclast and activating osteoblast. Biochem Biophys Res Commun. 2005;331(2):520-526. doi: https://doi.org/10.1016/j.bbrc.2005.03.210

15. Bollag RJ, Zhong Q, Phillips P, et al. Osteoblast-derived cells express functional glucose-dependent insulinotropic peptide receptors. Endocrinology. 2000;141(3):1228-1235. doi: https://doi.org/10.1210/endo.141.3.7366 
16. Zhong Q, Itokawa T, Sridhar S, et al. Effects of glucosedependent insulinotropic peptide on osteoclast function. Am J Physiol Endocrinol Metab. 2007;292(2):E543-548. doi: https://doi.org/10.1152/ajpendo.00364.2006

17. Бабенко А.Ю., Никитин В.С., Каронова ТЛ. Диабет и кость. Фокус на ингибиторы ДПП-4. // Медицинский Совет. — 2015. - №17. C. 108-113. [Babenko AY, Nikitin VS, Karonova TL. Diabetes and bone. Focus on DPP-4 inhibitors. Meditsinsky sovet. 2015;(17):108-113. (In Russ.)]

18. Rao RS, Kini S. GIP and bariatric surgery. Obes Surg. 2011;21(2):244-252. doi: https://doi.org/10.1007/s11695-010-0305-x

19. Fukushima N, Hanada R, Teranishi $\mathrm{H}$, et al. Ghrelin directly regulates bone formation. J Bone Miner Res. 2005;20(5):790-798. doi: https://doi.org/10.1359/JBMR.041237

20. Vincent RP, le Roux CW. Changes in gut hormones after bariatric surgery. Clin Endocrinol (Oxf). 2008;69(2):173-179. doi: https://doi.org/10.1111/j.1365-2265.2007.03164.x

21. Мелмед Ш., Полонски К.С., Ларсен П.Р., Кроненберг Г.М. Минеральный обмен. / Под ред. Дедова И.И., Мельниченко Г.А. М.: ГЭОТАР-Медиа, 2019. [Melmed S, Polonsky KS, Larsen PR, Cronenberg GM. Dedov II, Melnichenko GA, editors. Mineral'nyy obmen. Moscow: GEOTAR-Media; 2019. (In Russ.)]

22. Corbeels K, Verlinden L, Lannoo M, et al. Thin bones: Vitamin D and calcium handling after bariatric surgery. Bone Rep. 2018;8:57-63. doi: https://doi.org/10.1016/j.bonr.2018.02.002

23. Korner J, Inabnet W, Febres $G$, et al. Prospective study of gut hormone and metabolic changes after adjustable gastric banding and Roux-en-Y gastric bypass. Int J Obes (Lond). 2009;33(7):786-795. doi: https://doi.org/10.1038/ijo.2009.79

24. Winkler IG, Sims NA, Pettit AR, et al. Bone marrow macrophages maintain hematopoietic stem cell (HSC) niches and their depletion mobilizes HSCs. Blood. 2010;116(23):4815-4828. doi: https://doi.org/10.1182/blood-2009-11-253534

25. Carulli G. Effects of recombinant human granulocyte colonystimulating factor administration on neutrophil phenotype and functions. Haematologica. 1997;82(5):606-616.

26. Юдин В.А., Панфилова М.С. Особенности метаболизма костной ткани у пациентов с морбидным ожирением. // Хирургическая практика. - 2018. - № 1. - C. 65-68. [Yudin VA Panfilova MS. Bone metabolism features in patients with morbid obesity. Khirurgicheskaya praktika. 2018;(1):65-68. (In Russ.)] doi: https://doi.org/10.17238/issn2223-2427.2018.1.65-68

27. Бодунова Н.А., Аскерханов Р.Г., Хатьков И.Е, и др. Влияние бариатрических операций на обмен витаминов у больных ожирением. // Терапевтический архив. - 2015. - Т. 87. №2. - C. 70-76. [Bodunova NA, Askerkhanov RG, Khat'kov IE, et al. Impact of bariatric surgery on vitamin metabolisms in obese patients. Ter Arkh. 2015;87(2):70-76. (In Russ.)] doi: https://doi.org/10.17116/terarkh201587270-76

28. Мазурина Н.В., Огнева Н.А., Трошина Е.А., и др. Нарушения метаболизма кальция в отдаленном периоде после бариатрических операций. // Экспериментальная и клиническая гастроэнтерология. - 2013. - №12. - С. 27-32. [Mazurina NV, Ogneva NA, Troshina EA, et al. Abnormal calcium metabolism in the remote period after bariatric surgery. Experimental \& clinical gastroenterology. 2013;(12):27-32. (In Russ.)]

29. Березницкий Я.С., Дука Р.В. Характеристика изменений показателей липидного и углеводного обменов у больных морбидным ожирением до и после оперативного лечения в зависимости от вида хирургического вмешательства. // Гастроэнтерология. — 2018. - Т. 52. — №1. - C. 44-54. [Bereznitsky YS, Duka RV. Characteristics of changes in lipid and carbohydrate metabolism indices in patients with morbid obesity before and after surgical treatment depending on the type of surgical intervention. Gastroenterology. 2018;52(1):44-54. (In Russ.)] doi: https://doi.org/10.22141/2308-2097.52.1.2018.130777
30. Karefylakis C, Naslund I, Edholm D, et al. Vitamin D status 10 years after primary gastric bypass: gravely high prevalence of hypovitaminosis D and raised PTH levels. Obes Surg. 2014;24(3):343-348. doi: https://doi.org/10.1007/s11695-013-1104-y

31. Svanevik M, Risstad H, Hofso D, et al. Bone Turnover Markers After Standard and Distal Roux-en-Y Gastric Bypass: Results from a Randomized Controlled Trial. Obes Surg. 2019;29(9):2886-2895. doi: https://doi.org/10.1007/s11695-019-03909-1

32. Ivaska KK, Huovinen V, Soinio M, et al. Changes in bone metabolism after bariatric surgery by gastric bypass or sleeve gastrectomy. Bone. 2017:95:47-54. doi: https://doi.org/10.1016/j.bone.2016.11.001

33. Costa TL, Paganotto M, Radominski RB, et al. Calcium metabolism, vitamin $\mathrm{D}$ and bone mineral density after bariatric surgery. Osteoporos Int. 2015;26(2):757-764. doi: https://doi.org/10.1007/s00198-014-2962-4

34. Muller MK, Gero D, Reitnauer D, et al. The Impact of Roux-en-Y Gastric Bypass on Bone Remodeling Expressed by the P1NP/betaCTX Ratio: a Single-Center Prospective Cohort Study. Obes Surg. 2019;29(4):1185-1194. doi: https://doi.org/10.1007/s11695-018-03640-3

35. Scott D, Shore-Lorenti C, Ebeling PR. Multiple vertebral compression fractures after sleeve gastrectomy and a subsequent pregnancy: a case report. Osteoporos Int. 2019;30(10):2151-2154. doi: https://doi.org/10.1007/s00198-019-05073-9

36. Nypaver C, Bernstein J, Mehta S. Bilateral subtrochanteric femur insufficiency fractures after bariatric surgery: a case report. Osteoporos Int. 2019;30(6):1317-1320. doi: https://doi.org/10.1007/s00198-019-04956-1

37. Ghazi AA, Amirbaigloo A. Hypocalcemia and osteomalacia after bariatric surgery. Clin Cases Miner Bone Metab. 2017;14(2):227-229. doi: https://doi.org/10.11138/ccmbm/2017.14.1.227

38. Higgins SC, Papasavvas G. Multiple vertebral fractures sustained 5 months after Roux-en-Y gastric bypass: a case report. Arch Osteoporos. 2018;13(1):51. doi: https://doi.org/10.1007/s11657-018-0471-3

39. Goldner WS, Stoner JA, Lyden E, et al. Finding the optimal dose of vitamin D following Roux-en-Y gastric bypass: a prospective, randomized pilot clinical trial. Obes Surg. 2009;19(2):173-179. doi: https://doi.org/10.1007/s11695-008-9680-y

40. Carlin AM, Rao DS, Yager KM, et al. Treatment of vitamin D depletion after Roux-en-Y gastric bypass: a randomized prospective clinical trial. Surg Obes Relat Dis. 2009;5(4):444-449. doi: https://doi.org/10.1016/j.soard.2008.08.004

41. Luger M, Kruschitz R, Marculescu R, et al. The link between obesity and vitamin $D$ in bariatric patients with omega-loop gastric bypass surgery - a vitamin D supplementation trial to compare the efficacy of postoperative cholecalciferol loading (LOAD): study protocol for a randomized controlled trial. Trials. 2015;16:328. doi: https://doi.org/10.1186/s13063-015-0877-9

42. Luger M, Kruschitz R, Kienbacher C, et al. Vitamin D3 Loading Is Superior to Conventional Supplementation After Weight Loss Surgery in Vitamin D-Deficient Morbidly Obese Patients: a Double-Blind Randomized Placebo-Controlled Trial. Obes Surg. 2017;27(5):1196-1207. doi: https://doi.org/10.1007/s11695-016-2437-0

43. Smelt HJM, Pouwels S, Smulders JF. The Influence of Different Cholecalciferol Supplementation Regimes on 25(OH) Cholecalciferol, Calcium and Parathyroid Hormone after Bariatric Surgery. Medicina (Kaunas). 2019;55(6). doi: https://doi.org/10.3390/medicina55060252

44. Cole AJ, Beckman LM, Earthman CP. Vitamin D status following bariatric surgery: implications and recommendations. Nutr Clin Pract. 2014:29(6):751-758. doi: https://doi.org/10.1177/0884533614546888

45. Muschitz C, Kocijan R, Haschka J, et al. The Impact of Vitamin D, Calcium, Protein Supplementation, and Physical Exercise on Bone Metabolism After Bariatric Surgery: The BABS Study. J Bone Miner Res. 2016;31 (3):672-682. doi: https://doi.org/10.1002/jbmr.2707

ИНФОРМАЦИЯ ОБ АВТОРАХ [AUTHORS INFO]:

*Берковская Марина Ароновна, к.м.н. [Marina A. Berkovskaya, MD, PhD]; адрес: Россия, 119435, Москва, ул. Погодинская, д.1 [address: 1 Pogodinskaya street, 119435 Moscow, Russia]; ORCID: https://orcid.org/0000-0003-4974-7765; eLibrary SPIN: 4251-7117; e-mail: abaitamar@gmail.com 
Кушханашхова Дана Аслановна, аспирант [Dana A. Kushkhanashkhova, postgraduate student];

ORCID: https://orcid.org/0000-0003-1598-624X; e-mail: dana.kuch@mail.ru

Сыч Юлия Петровна, к.M.H. [Yulia P. Sych, MD, PhD]; ORCID: https://orcid.org/0000-0002-7000-0095;

eLibrary SPIN: 3406-0978; e-mail: juliasytch@mail.ru

Фадеев Валентин Викторович, д.м.н., профессор, член-корреспондент РАH [Valentin V. Fadeyev, MD, ScD, professor]; ORCID: https://orcid.org/0000-0002-3026-6315; eLibrary SPIN: 6825-8417; e-mail: walfad@mail.ru

*Автор, ответственный за переписку / Corresponding author.

\section{ЦИТИРОВАТЬ:}

Берковская М.А., Кушханашхова Д.А., Сыч Ю.П., Фадеев В.В. Состояние фосфорно-кальциевого обмена у пациентов после бариатрических операций и роль восполнения дефицита витамина D в профилактике и лечении послеоперационных костно-метаболических нарушений // Ожирение и метаболизм. - 2020. - Т. 17. - №1. - С. 73-81. doi: https://doi.org/10.14341/omet12306

\section{TO CITE THIS ARTICLE:}

Berkovskaya MA, Kushkhanashkhova DA, Sych YP, Fadeyev VV. Characteristics of calcium and phosphorous metabolism in patients after bariatric surgery and the role of vitamin D supplementation in the prevention and treatment of postoperative bone and mineral disorders. Obesity and metabolism. 2020;17(1):73-81. doi: https://doi.org/10.14341/omet12306 